\title{
Clinical Features of a Large-Area Cerebral Infarction With Good Prognosis
}

\author{
Yi Bao ${ }^{\mathrm{a}, \mathrm{b}}$, Yayong Ding ${ }^{\mathrm{a}}$, Quanying Liu ${ }^{\mathrm{a}}$, Cong $\mathrm{Wu}^{\mathrm{a}}$, Lingge $\mathrm{Xu}^{\mathrm{a}}$, \\ Xiaodong $\mathrm{Liu}^{\mathrm{a}}$, Guangjian $\mathrm{Liu}^{\mathrm{a}}$
}

\begin{abstract}
Large-area cerebral infarction has a high disability rate, and is a serious threat to patients' lives. This article reports that a patient with a large-area cerebral infarction with a midline shift had recovered well after conservative treatment, and analyzes the reasons. We described the onset of the disease, the course of treatment, the evolution of the disease and response measures, combined with imaging to show the progress and changes of the disease. Although there was no thrombolysis, the blood vessels were recanalized. When the midline was displaced, although the bone flap was not removed to reduce the intracranial pressure, cerebral hernia was effectively prevented by dehydration treatment. The patient had severe loss of nerve function at the onset of the disease. After comprehensive treatment, good results have been achieved. Comprehensive treatment in stroke unit is helpful to save ischemic penumbra. Close monitoring of patients' vital signs and mental changes is conducive to timely response to changes in the disease. Early rehabilitation is conducive to the recovery of neurological function. For the case of low thrombolytic acceptance rate, screening before thrombolysis, close observation during thrombolysis and timely reviewing after thrombolysis are adopted to actively respond to hemorrhage transformation after thrombolysis, which improve the safety and acceptability of treatment.
\end{abstract}

Keywords: Cerebral infarction; Digital subtraction angiography; Hemorrhage transformation; Thrombolysis; NIHSS score

\section{Introduction}

Acute ischemic stroke is characterized by high morbidity, recurrence rate, multiple complications, disability rate and mortality. It accounts for $70-80 \%$ of strokes, and commonly occurs in middle-aged and elderly people [1-3].

Manuscript submitted April 14, 2019, accepted April 29, 2019

aDepartment of Neurology, Taihe Hospital Affiliated to Hubei University of Medicine, Shiyan City, Hubei Province, China

${ }^{b}$ Corresponding Author: Yi Bao, Department of Neurology, Taihe Hospital Affiliated to Hubei University of Medicine, Shiyan City, Hubei Province, China. Email:karlbaoyi@163.com

doi: https://doi.org/10.14740/jmc3298
Acute cerebral infarction leads to cerebral tissue ischemia, hypoxia and necrosis, mainly manifested as a series of neurological deficits such as disturbance of consciousness, aphasia and hemiplegia. Internal carotid artery occlusion can lead to contralateral side: hemianopia, hemiplegia, partial sensory disturbance and aphasia in dominant hemisphere. Blood supply areas of vertebral-basal artery include occipital lobe, thalamus, corpus callosum, cerebellum and brainstem. When a cerebral infarction occurs, especially in the brain stem where the nerve fiber bundles in the respiratory and circulatory centers are concentrated, if the treatment is not treated in time, the mortality rate is as high as $80-90 \%$, which seriously threatens the life of the patient $[4,5]$.

This article reports a patient with a large-area cerebral infarction. The level of consciousness was significantly reduced at the onset, the tone of the speech was not clear, the limb muscle strength was significantly reduced, there was confusion in the course of the disease and magnetic resonance imaging (MRI) showed that the midline shift was obvious. After drug treatment, the patient's condition recovered well.

\section{Case Report}

A 55-year-old man was admitted to the hospital due to unclear speech and left limb weakness for $4 \mathrm{~h}$. The symptoms were left limb weakness, inability to stand and walk, inability to hold objects in the left hand, accompanied by unclear speech, no obvious headache, no dizziness, no nausea and vomiting, without disorder of consciousness, no coughing caused by drinking water, no feces and urine incontinence and body convulsions. He had a history of hyperlipidemia, a long history of heavy smoking and no history of hypertension, diabetes or heart disease. Physical examination showed temperature of $36.6{ }^{\circ} \mathrm{C}$, pulse of 78 beats/min, respiratory rate of 13 beats $/ \mathrm{min}$ and blood pressure of $156 / 87 \mathrm{~mm} \mathrm{Hg}$. The mind was lethargic, and the examination was not cooperative. No obvious abnormality was found in physical examination of internal medicine system. Neurological examination showed both pupils were equally round, diameter $3 \mathrm{~mm}$, sensitive to light reflection, eyes gazed to the right, no nystagmus, left nasolabial fold shallow, tongue extended to the left, unclear spit, right side lower extremity limb muscle strength 5, left upper and lower limb muscle strength 3 , limb muscle tension was normal, sensory physical examination did not cooperate, bilateral sacral reflex was equal, left Babinski sign was positive, neck was soft and 

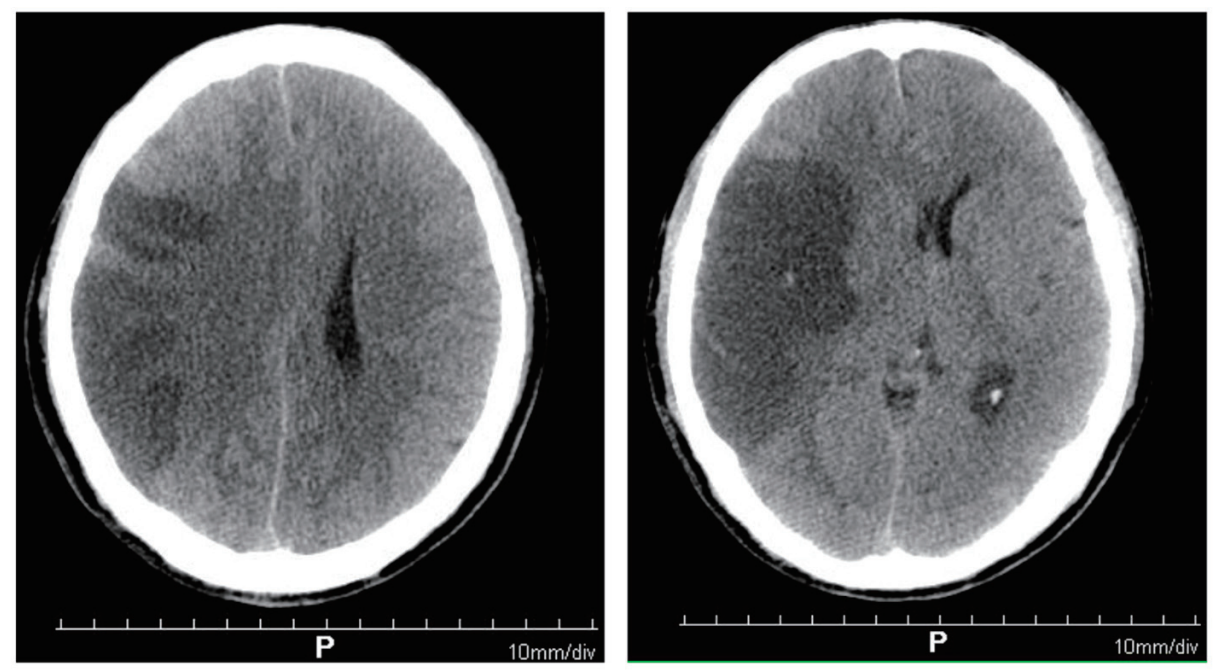

Figure 1. Brain computed tomography showing large-area cerebral infarction in the right frontal parietal lobe (the blood supply area of the middle cerebral artery).

non-resistance, and meninges irritation was negative. Emergency head computed tomography (CT) showed no bleeding.

In diagnosis analysis, the patient presented with binocular gazed to the right, and the left limb was hemiplegic, localization in the right cerebral hemisphere, basal ganglia area. Qualitative analysis showed elderly man with basic medical history of hyperlipidemia, a long history of heavy smoking, acute onset, left limb weakness and unclear speech, and physical examination showed the patient's left limb muscle strength was level 3. There were no headaches and other whole brain symptoms, no meningeal irritation, no bleeding in the head CT report, and cerebral infarction was considered as the first diagnosis. National Institutes of Health stroke scale (NIHSS) score was 9 points and TOAST classification was aortic atherosclerosis.

In treatment course, the patient had normal blood routine, blood coagulation function and blood glucose, and was currently in the thrombolysis time window of acute cerebral infarction, with indications for emergency thrombolysis and no obvious contraindications. Recombinant tissue plasminogen activator (rt-PA) thrombolytic therapy was suggested for the patients and their families, and bridging therapy was suggested if necessary, but the patients' families refused. Antiplatelet aggregation ( $0.1 \mathrm{~g}$ of aspirin, once a day), enhanced lipid-lowering (20 $\mathrm{mg}$ of rosuvastatin, once a night), improving microcirculation, scavenging of oxygen free radicals (30 $\mathrm{mg}$ of edaravone, twice a day), expansion of blood volume and establishment of collateral circulation ( $0.2 \mathrm{~g}$ of butanopeptide, three times a day) were given. In addition, acupuncture and neuromuscular intermediate frequency pulse therapy were used. After $24 \mathrm{~h}$ of onset, the CT scan of the brain showed a large area of cerebral infarction in the right frontal lobe (the middle cerebral artery supply area) (Fig. 1). Mannitol (125 $\mathrm{mL}$, once every $8 \mathrm{~h}$ ) was added to reduce intracranial pressure to prevent brain edema.

On the third day after the onset, the patient was comatose. In brain MRI, diffusion-weighted imaging (DWI) showed that there were multiple plaque-like and cerebral gyrus signals on the right occipital lobe, but no obvious abnormal high-signal lesions were found in the remaining cranial. Acute infarction of the right occipital lobe was found. There was acute infarction of the right frontotemporal parietal lobe (Fig. 2). The midline displacement of large-area cerebral infarction in the patient had the risk of cerebral herniation, which was lifethreatening. A neurosurgical consultation was requested and decompression of the bone flap was recommended, but the patient's family refused. The dehydration regimen was adjusted as mannitol (125 mL, once every $6 \mathrm{~h})$, glycerin fructose $(250$ $\mathrm{mL}$ twice daily) and furosemide (20 $\mathrm{mg}$ twice daily) to prevent cerebral palsy formation. We paid attention to the balance of internal environment, and closely observed the patient's mental changes. The narcolepsy of the patient gradually decreased from the seventh day of the onset.

On the 10th day of onset, the patient was conscious and the muscle tension was normal. NIHSS score was 0 points. Reexamination of craniocerebral CT showed that the right frontotemporal parietal lobe infarction was less severe than before (Fig. 3). Systemic heparinization was performed to ascertain the cerebrovascular status of the patient, right radial artery puncture, sheath insertion and intubation were performed with Seldinger technique, and catheters were selectively inserted into the aortic arch, bilateral common carotid artery, subclavian internal carotid artery and vertebral artery for digital subtraction angiography (DSA) examination. The results showed no abnormalities in the patient's cerebrovascular (Fig. 4).

\section{Discussion}

The incidence of acute cerebral infarction is increasing with the increase of population aging and lifestyle change. According to statistics, the annual incidence rate in China alone was $120 / 100,000$ to $180 / 100,000$, and the mortality rate was $60 / 100,000$ to $120 / 100,000$. What's more serious is that the 

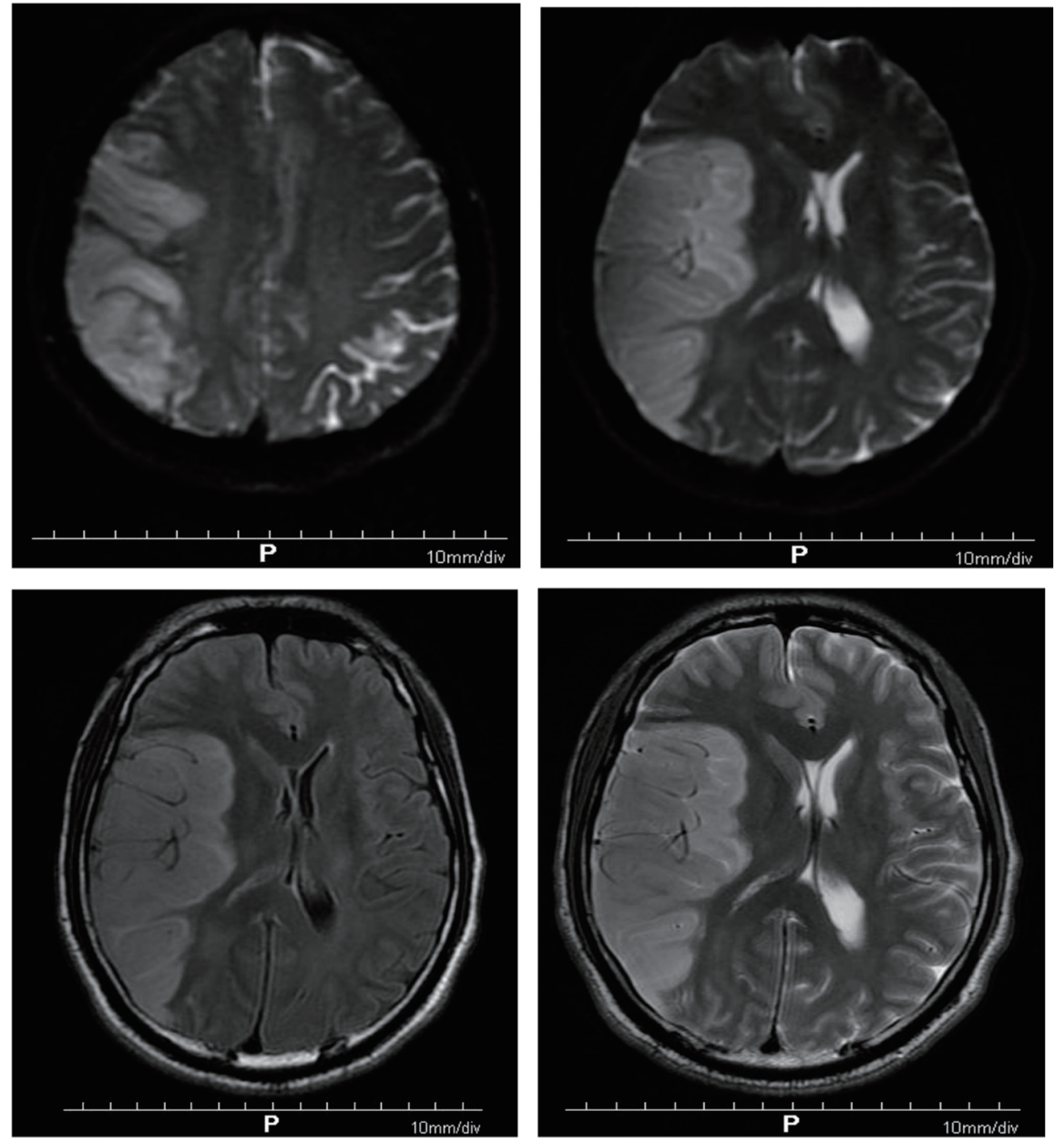

Figure 2. Diffusion-weighted imaging showing multiple patchy and gyriform hyperintensity signals in the right temporoparietal occipital lobe, but no obvious abnormal hyperintensity lesions in the rest of the skull. Acute infarction of the right temporoparietal occipital lobe was found. Brain MRI showing acute infarction of right frontotemporal parietal lobe.
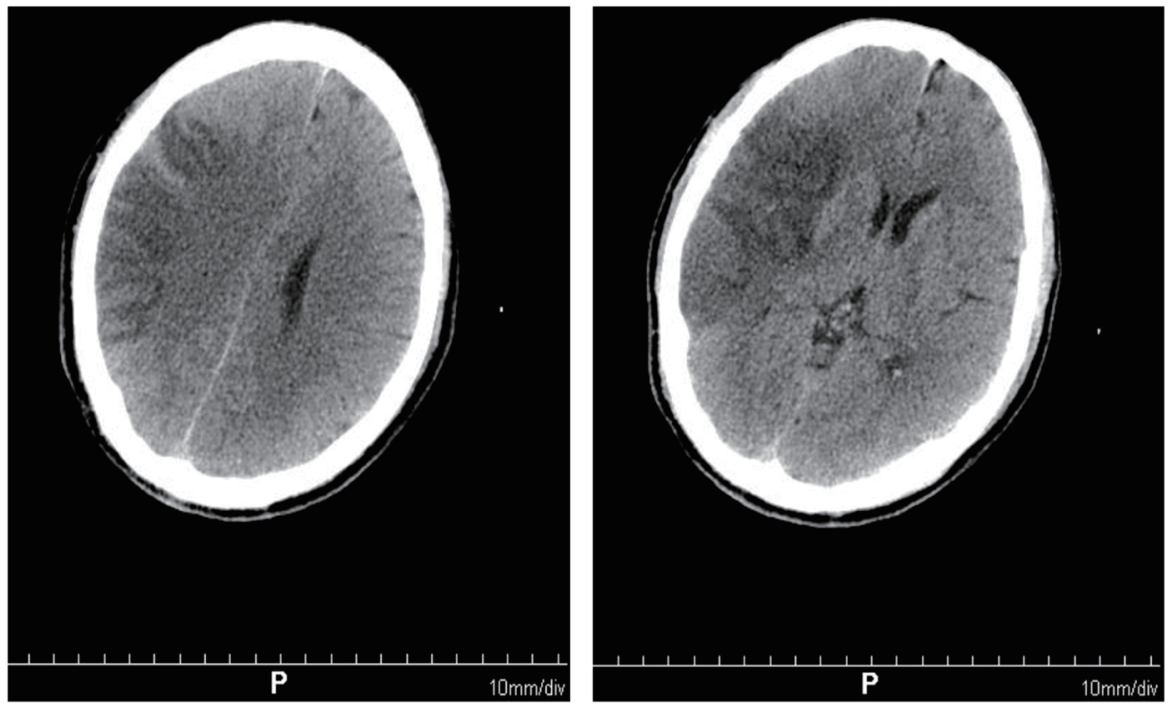

Figure 3. Brain computed tomography scan showing right frontal temporal parietal lobe infarction, less severe than before. 


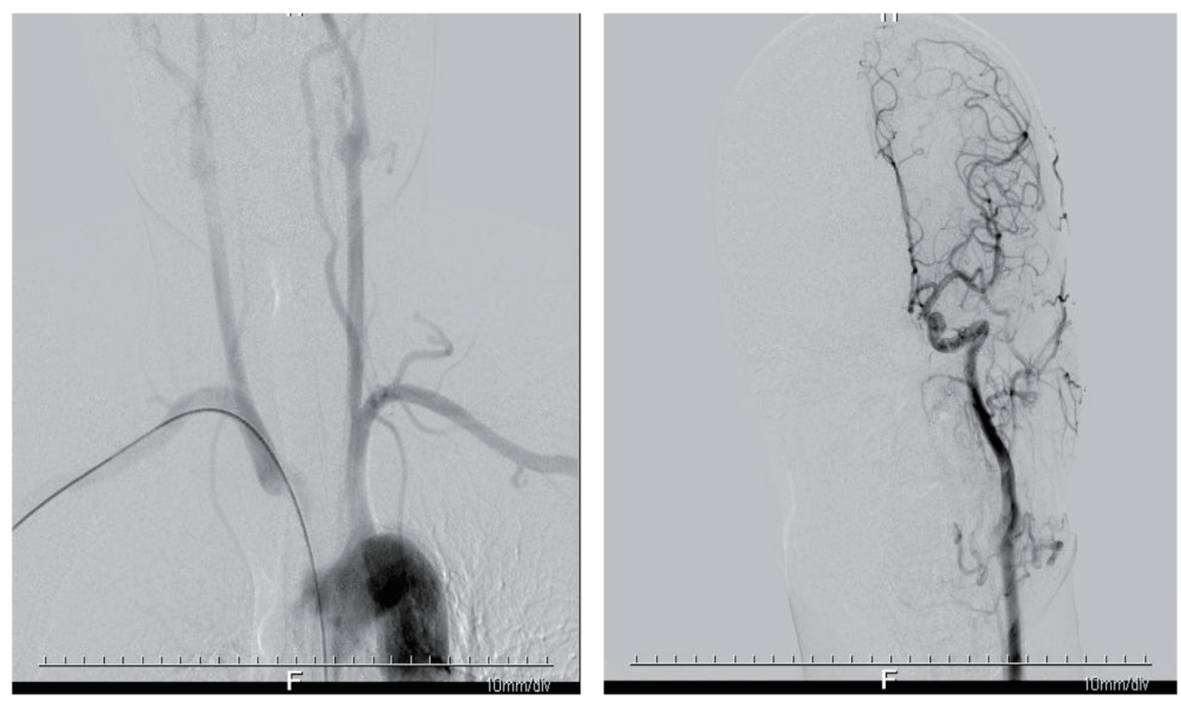

Figure 4. Digital subtraction angiography showing no significant stenosis and infarction in the patient's cerebrovascular.

age of onset tends to be younger in recent years [6-8]. The patient was a middle-aged man, with a risk factor for high blood lipids and long-term smoking, and was a high-risk group of cerebral infarction.

There is almost no reserve of glucose and oxygen in the brain tissue, which is very sensitive to ischemia and hypoxia. A constant supply of blood must be available to maintain the normal nerve function of the brain tissue. In the case of acute ischemia, the pathophysiological mechanism of neuronal necrosis is very complex, mainly involving energy deficiency, intracellular $\mathrm{Ca}^{2+}$ overload, ion imbalance, cytokine release, activation of neurotoxic free radicals, inflammatory response, blood-brain barrier disruption, etc., and the symptoms are acute loss of neurological function $[9,10]$. The patient's acute onset, significantly decreased level of consciousness, unclear speech, obvious decrease in left limb muscle strength and fuzzy consciousness in the course of the disease were consistent with the clinical manifestations of acute cerebrovascular disease.

The key to rescue ischemic penumbra is to restore blood perfusion in time. Early intravenous thrombolysis and bridging treatment can rescue brain tissue between electrical failure and membrane failure [11-13]. The current strategies for rescue of ischemic penumbra are: intravenous thrombolysis (IVT), arterial thrombolysis (IAT), arterial and venous combined thrombolysis ICT + IAT, balloon dilatation, microcatheter and microwire thrombolysis, thrombectomy, stent-assisted thrombolysis, ultrasound-assisted thrombolysis and a combination of these technologies [14-17]. However, the patient and his family refused thrombolysis, and the reasons were lack of understanding of the safety and effectiveness of thrombolysis, especially hemorrhagic transformation. Comprehensive treatment in the stroke unit (SU) includes anti-platelet aggregation therapy, anticoagulation, fibrinolysis, enhanced lipid-lowering, dilatation, vasodilation, improvement of microcirculation, removal of oxygen free radicals, establishment of collateral circulation and prevention of complications. The patient timely adopted comprehensive treatment such as anti-platelet ag- gregation and establishment of collateral circulation. When the level of consciousness decreased, timely imaging examination was done to rule out hemorrhagic transformation, although no decompression of bone flap was performed, dehydration was strengthened in time to effectively prevent the formation of cerebral hernia.

There are some reasons for the patient's good recovery. 1) Although no intravenous thrombolytic therapy was performed, comprehensive treatment in the SU effectively controlled the patient's condition, combined with the fact that no obvious infarction was found in the cerebral angiography of the patient, and it was considered that the newly generated thrombus might still dissolve in the case of anti-platelet therapy. 2) Recanalization of blood vessels timely restored blood perfusion and effectively saved ischemic penumbra, which could be shown in Figures 1 and 3. In the aspect of establishing collateral circulation, butylphthalide can promote the establishment of collateral circulation around the ischemic area, increase the velocity of arterial blood flow and play a role in increasing the blood supply in the ischemic area. At the same time, it can improve the stability of mitochondrial membrane in the cell, enhance the ATPase activity in the mitochondria, stabilize the mitochondrial structure and play a role in reducing neuron cell death. 3) The patient's vital signs and mental changes were closely monitored, and the patient's consciousness level was found to be decreased in time, and positive countermeasures were taken to avoid the formation of cerebral hernia. 4) Early acupuncture and neuromuscular therapy were conducive to the recovery of nerve function.

Shortcomings and limitations of this case were that this case is a single clinical case, and although without thrombolysis, the patient's blood vessel was recanalized. Although no decompression of bone flap was performed during midline displacement, cerebral hernia was effectively prevented by dehydration treatment. Patient with severe neurological deficits at the onset of disease, after comprehensive treatment, achieved good results. Nevertheless, a single case is not enough to dem- 
onstrate the safety and effectiveness of conservative treatment, which requires big data analysis and support.

Ischemic stroke leads to cerebral tissue ischemia, hypoxia and necrosis, which is characterized by high morbidity, mortality, disability, recurrence rate and multiple complications. Cerebral vascular occlusion does not immediately lead to cerebral infarction, and the specific development trend is closely related to the severity of ischemia and ischemic time. Comprehensive treatment in SU is helpful to save ischemic penumbra. Close monitoring of patients' vital signs and mental changes is conducive to timely response to changes in the disease. Early rehabilitation is conducive to the recovery of neurological function. In view of the low acceptance rate of thrombolysis, screening before thrombolysis, close observation during thrombolysis and timely reviewing after thrombolysis are adopted to actively respond to hemorrhagic transformation after thrombolysis, which improve the safety and acceptability of treatment.

\section{Acknowledgments}

We are grateful to the medical staff who have treated this patient carefully.

\section{Funding Disclosure}

This is a clinical observation article without any funding.

\section{Conflict of Interest}

The authors declare that they have no conflict of interest.

\section{Informed Consent}

We obtained the patient's informed consent.

\section{Author Contributions}

Yi Bao summed up and wrote the article; Yayong Ding and Quanying Liu wrote the article; Cong Wu, Lingge Xu, Xiaodong Liu and Guangjian Liu collected the case data.

\section{References}

1. Pan A, Sun Q, Okereke OI, Rexrode KM, Hu FB. Depression and risk of stroke morbidity and mortality: a meta-analysis and systematic review. JAMA. 2011;306(11):1241-1249.

2. Favate AS, Younger DS. Epidemiology of Ischemic Stroke. Neurol Clin. 2016;34(4):967-980.

3. Truelsen T, Krarup LH, Iversen HK, Mensah GA, Feigin
VL, Sposato LA, Naghavi M. Causes of death data in the global burden of disease estimates for ischemic and hemorrhagic stroke. Neuroepidemiology. 2015;45(3):152160.

4. Paciaroni M, Balucani C, Agnelli G, Caso V, Silvestrelli G, Grotta JC, Demchuk AM, et al. Systemic thrombolysis in patients with acute ischemic stroke and Internal Carotid ARtery Occlusion: the ICARO study. Stroke. 2012;43(1):125-130.

5. Fukuoka T, Nakazato Y, Kawasaki H, Ikeda K, Furuya T, Miyake A, Mitsufuji T, et al. The clinical features of ischemic stroke patients for whom smoking was considered the sole risk factor for ischemic stroke. Intern Med. 2018;57(12):1703-1706.

6. Colivicchi F, Bassi A, Santini M, Caltagirone C. Discontinuation of statin therapy and clinical outcome after ischemic stroke. Stroke. 2007;38(10):2652-2657.

7. Ji R, Schwamm LH, Pervez MA, Singhal AB. Ischemic stroke and transient ischemic attack in young adults: risk factors, diagnostic yield, neuroimaging, and thrombolysis. JAMA Neurol. 2013;70(1):51-57.

8. Putaala J, Metso TM, Metso AJ, Makela E, Haapaniemi E, Salonen O, Kaste M, et al. Thrombolysis in young adults with ischemic stroke. Stroke. 2009;40(6):20852091.

9. Vannucci RC, Vannucci SJ. Glucose, acidosis, and perinatal hypoxic - ischemic brain damage. Developmental Disabilities Research Reviews. 2015;3(1):69-75.

10. Seifert G, Schilling K, Steinhauser C. Astrocyte dysfunction in neurological disorders: a molecular perspective. Nat Rev Neurosci. 2006;7(3):194-206.

11. Zerna C, Thomalla G, Campbell BCV, Rha JH, Hill MD. Current practice and future directions in the diagnosis and acute treatment of ischaemic stroke. Lancet. 2018;392(10154):1247-1256.

12. Saver JL. Time is brain-quantified. Stroke. 2006;37(1):263-266.

13. Audebert HJ, Sobesky J. Stroke: 'time is brain' after stroke, regardless of age and severity. Nat Rev Neurol. 2014;10(12):675-676.

14. Donnan GA, Davis SM. Neuroimaging, the ischaemic penumbra, and selection of patients for acute stroke therapy. Lancet Neurol. 2002;1(7):417-425.

15. Awadh M, MacDougall N, Santosh C, Teasdale E, Baird T, Muir KW. Early recurrent ischemic stroke complicating intravenous thrombolysis for stroke: incidence and association with atrial fibrillation. Stroke. 2010;41(9):19901995.

16. Kim JS, Kim YJ, Lee KB, Cha JK, Park JM, Hwang Y, Kim EG, et al. Low- versus standard-dose intravenous alteplase in the context of bridging therapy for acute ischemic stroke: a Korean ENCHANTED study. J Stroke. 2018;20(1):131-139.

17. Gerschenfeld G, Muresan IP, Blanc R, Obadia M, Abrivard M, Piotin M, Alamowitch S. Two paradigms for endovascular thrombectomy after intravenous thrombolysis for acute ischemic stroke. JAMA Neurol. 2017;74(5):549556. 